\title{
A Moderate Algorithm for Generalized Radial basis Function Neural Networks
}

\author{
B.M.Singhal \\ Department of Computer Application, ITM - Universe, Gwalior, India.
}

\begin{abstract}
A Radial Basis Function (RBF) neural network can be regarded as a feed forward network composed of multiple layers of neurons with entirely different roles. The input layer made of sensory units that connect the network to its environment. A radial basis function neural network depends mainly upon an adequate choice of the number and positions of its basis function centers. In case of generalized RBF neural network, the output layer is linear and supplying each layer response as the linear combination of the hidden responses. In this paper we have proposed a moderate algorithm for most generalized form of RBF neural network and the results may be reduced for various forms of $\mathrm{RBF}$ and other artificial neural networks as particular cases.
\end{abstract}

Key Words - Radial Basis Function, Neural Networks.

\section{INTRODUCTION}

The radial basis function neural networks are powerful function approximators for multivariate nonlinear continuous mappings. They have a simple architecture and the learning algorithm corresponds to the solution of a linear regression problem. The RBF network behavior strongly depends upon the number and the position of the basis functions at the hidden layer. Traditional method of determining the centers are randomly choose input vectors from the training data set or to obtain vectors from unsupervised clustering algorithms applied to input data or vectors obtain through a supervised learning scheme. In this paper we have proposed a moderate algorithm for most generalized RBF neural network and the results may be reduced for various forms of RBF and other artificial neural networks as particular cases.

Let $x_{11}, \ldots, x_{1 r_{1}}$ is a set of inputs and $c_{11}, \ldots, c_{1 r_{1}}$ be the set of centers, then

$$
\left\|x_{1 k}-c_{1 k}\right\|_{;} \mathrm{k}=1,2, \ldots, r_{1}
$$

be the radial vectors in Euclidean sense. If the corresponding weights are

$$
w_{1 k} \quad ; \mathrm{k}=1,2, \ldots, r_{1}
$$

the radial strength of $x_{1 k}$ will be

$$
w_{1 k}\left\|x_{1 k}-c_{1 k}\right\| \quad ; \mathrm{k}=1,2, \ldots, r_{1}
$$

Then the combined probabilistic strength of type $x_{1 k} \quad$ will be

$$
\prod_{k=1}^{r_{1}} \frac{\left\{w_{1 k}\left\|x_{1 k}-c_{1 k}\right\|\right\}^{m_{1 k}}}{m_{1 k} !}
$$

for $m_{1 k}$ external input lines and $\mathrm{n}$ units of RBF neural network.
If we choose an activation function

$$
f_{1}\left(\sum_{k=1}^{r_{1}} m_{1 k}\right)
$$

the system output for first stage of generalized RBF neural network with the weight vector

$$
\mathrm{W}=\left\{w_{11}, w_{12}, \ldots, w_{1 r_{1}}\right\}
$$

may be taken as $\left(0 \leq w_{1 i} \leq 1\right)$

$$
f_{1}\left(\sum_{k=1}^{r_{1}} m_{1 k}\right) \prod_{k=1}^{r_{1}} \frac{\left\{w_{1 k}\left\|x_{1 k}-c_{1 k}\right\|\right\}^{m_{1 k}}}{m_{1 k} !}
$$

Similarly for other stages of generalized RBF neural network, we have the outputs respectively as

$$
\begin{gathered}
f_{2}\left(\sum_{k=1}^{r_{2}} m_{2 k}\right) \prod_{k=1}^{r_{2}} \frac{\left(w_{2 k}\left\|x_{2 k}-c_{2 k}\right\|\right)^{m_{2 k}}}{m_{2 k} !} \\
\quad f_{n}\left(\sum_{k=1}^{r_{s}} m_{n k}\right) \prod_{k=1}^{r_{s}} \frac{\left(w_{n k}\left\|x_{n k}-c_{n k}\right\|\right)^{m_{n k}}}{m_{n k} !}
\end{gathered}
$$

Finally, the total output $\mathrm{Z}$ of generalized multilayer RBF neural network with an unified activation function

$$
f\left(\sum_{k=1}^{r_{1}} m_{1 k}+\ldots+\sum_{k=1}^{r_{s}} m_{n k}\right)
$$

may be taken as below (Because in case of indefinite large numbers the probabilistic models are best suited for infinity )

$$
\begin{gathered}
\sum_{\left(m_{1 r}\right)=0}^{\infty} \ldots \sum_{\left(m_{n s}\right)=0}^{\infty} \\
f\left(\sum_{k=1}^{r_{1}} m_{1 k}+\ldots+\sum_{k=1}^{r_{s}} m_{n k}\right) \\
f_{1}\left(\sum_{k=1}^{r_{1}} m_{1 k}\right) \quad f_{n}\left(\sum_{k=1}^{r_{s}} m_{n k}\right) \\
\prod_{k=1}^{r_{1}} \frac{\left(w_{1 k}\left\|x_{1 k}-c_{1 k}\right\|\right)^{m_{1 k}}}{m_{1 k} !} \mathrm{X} \\
\ldots \prod_{k=1}^{r_{s}} \frac{\left(w_{n k}\left\|x_{n k}-c_{n k}\right\|\right)^{m_{n k}}}{m_{n k} !} \\
\sum_{m_{1}, m_{2}, \ldots, m_{n}=0}^{\infty} f\left(m_{1}+m_{2}+\ldots+m_{n}\right)
\end{gathered}
$$




$$
\begin{array}{r}
\prod_{i=1}^{n} f_{i}\left(m_{i}\right) \frac{\left(\sum_{j=1}^{r_{1}} w_{1 j}\left\|x_{1 j}-c_{1 j}\right\|\right)^{m_{1}}}{m_{1} !} \\
\frac{\left(\sum_{j=1}^{r_{s}} w_{n j}\left\|x_{n j}-c_{n j}\right\|\right)^{m_{n}}}{m_{n} !}
\end{array}
$$

by using the Identity [9].

$$
\begin{aligned}
& \sum_{m_{1}, m_{2}, \ldots, m_{n}=0}^{\infty} f\left(m_{1}+m_{2}+\ldots+m_{n}\right) \prod_{i=1}^{n} f_{i}\left(m_{i}\right) \\
& \frac{\left(\sum_{j=1}^{r_{1}} x_{1 j}\right)^{m_{1}}}{m_{1} !} \quad \ldots \quad \frac{\left(\sum_{j=1}^{r_{s}} x_{n j}\right)^{m_{n}}}{m_{n} !} \\
& \sum_{\left(m_{1 r}\right)=0}^{\infty} \cdots \sum_{\left(m_{n s}\right)=0}^{\infty} \\
& f\left(\sum_{k=1}^{r_{1}} m_{1 k}+\ldots+\sum_{k=1}^{r_{s}} m_{n k}\right) \\
& f_{1}\left(\sum_{k=1}^{r_{1}} m_{1 k}\right) \quad f_{n}\left(\sum_{k=1}^{r_{s}} m_{n k}\right) \\
& \mathrm{X} \prod_{k=1}^{r_{1}} \frac{\left(x_{1 k}\right)^{m_{1 k}}}{m_{1 k} !} \prod_{k=1}^{r_{s}} \frac{\left(x_{n k}\right)^{m_{n k}}}{m_{n k} !} \\
& f\left(\sum_{i=1}^{n} m_{i}\right)
\end{aligned}
$$$$
\text { In case of symmetrical layers }
$$

may be taken as constant say, $\delta$. Also a group of

in R.H.S. of (1) neurons are similar in nature so $f_{i}\left(m_{i}\right)$ may be taken as modular parameters $\lambda_{i}^{m_{i}}$, say. So we have

$$
\begin{gathered}
\sum_{\mathrm{Z}_{1}, m_{2}, \ldots, m_{n}=0}^{\infty} \frac{\left(\lambda_{1} \sum_{j=1}^{r_{1}} w_{1 j}\left\|x_{1 j}-c_{1 j}\right\|\right)^{m_{1}}}{m_{1} !} \\
\left.\frac{\left(\lambda_{n} \sum_{j=1}^{r_{s}} w_{n j}\left\|x_{n j}-c_{n j}\right\|\right)^{m_{n}}}{m_{n} !}\right\} \\
=\delta \operatorname{Exp}\left(\lambda_{j=1}^{r_{1}} w_{1 j}\left\|x_{1 j}-c_{1 j}\right\|+\ldots\right.
\end{gathered}
$$

$$
\begin{array}{r}
\left.\lambda_{n} \sum_{j=1}^{r_{s}} w_{n j}\left\|x_{n j}-c_{n j}\right\|\right) \\
\ldots+\lambda_{1} \sum_{j=1}^{r_{1}} w_{1 j}\left\|x_{1 j}-c_{1 j}\right\|+\ldots \\
\propto \operatorname{Exp}\left(\lambda_{n} \sum_{j=1}^{r_{s}} w_{n j}\left\|x_{n j}-c_{n j}\right\|\right) \\
\ldots++
\end{array}
$$

That is in generalized RBF neural network, the out put is directly proportional to exponentially weighted sum of inputs and middle stages outcomes taking radially along with constraints $\lambda_{1}, \ldots, \lambda_{n}$; which may be suitably defined as per network configuration.

Particularly when we shift centers to origin, we have

$$
\begin{aligned}
& \lambda_{1} \sum_{j=1}^{r_{1}} w_{1 j} x_{1 j}+\ldots \\
& \text { Or } \\
& \left.\lambda_{n} \sum_{j=1}^{r_{s}} w_{n j} x_{n j}\right) \\
& \begin{array}{c}
\mathrm{Z} \propto \operatorname{Exp}\left(\lambda_{1} W_{1} X_{1}+\ldots+\lambda_{n} W_{n} X_{n}\right) \\
W_{i} X_{i}=\sum_{j=1}^{r_{i}} w_{i j} x_{i j}
\end{array}
\end{aligned}
$$

Also if the network configures with $\lambda_{i}=1 ; \mathrm{i}=1,2, \ldots, \mathrm{n}$ : then

We have

$\mathrm{Z} \propto \operatorname{Exp}\left(W_{1} X_{1}+\ldots+W_{n} X_{n}\right)$

Which resembles with generalized artificial neural network, and is the generalization of the author's result [10]

$$
\mathrm{Z} \propto \operatorname{Exp}(\mathrm{WX})
$$

\section{CONCLUSION}

In this paper we have proposed a moderate algorithm for most generalized RBF neural network, further work on application is being carried out and some fruitful results are expected in near future.

\section{REFERENCES}

[1]. J.Moody and C. Darken, "Fast learning in Networks of locally- tuned Processing units, Neural Computation , 1:281-294, 1989

[2]. N.B.Karayiannis and G.W. Mi, "Growing radial basis Neural Networks: merging supervised and unsupervised learning with network growth techniques " IEEE Trans. On Neural Networks.

[3]. D.Dasgupta and S. Forest, "Artificial Immune System in Industrial Applications” Proc. Of the IPMM’99, 1999. 
[4]. P.Hajela and J.S.Yoo, "Immune Network Modeling in design Optimization ".In new Ideas in Optimization,(Eds) D Corne, M.Dorigo \& F. Glover, McGraw Hill, London, pp. 203-215, 1999.

[5]. L.N.De Castro and F.J.Von Zuben, "An Evolutionary Immune Network for data clustering ", Proc. Of the IEEE Brazelian Symposium on Neural Networks, pp. 84$89,2000 \mathrm{~b}$.

[6]. D.S.Broomhead and D.Lowe, "Multivariate functional Interpolation and adaptive Networks", Complex Systems, 2:321-355, 1988.

[7]. M.J.D. Powell, "Radial Basis Functions for multivariable Interpolation", A reviw in IMA Conference, Algorithm for Appr. Of Functions and Data, J.C. Mason \& M.G. Cox (eds.), Oxford , U.K.: Oxford Univ. Press, 143-167, 1987.
[8]. C.A. Michelli, "Interpolation of Scattered Data: Distance Matrices and conditionally Positive definite Functions", Const.Approx.,2: 11-22, 1986.

[9]. B.M. Singhal and B.M. Agrawal, "On Multiple Integrals Involving Hypergeometric Functions of two Variables", Jnanabha Sect. A. Vol. 4, July 1974.

[10]. B.M. Singhal, "A proposed Algorithm for Multivariate Artificial Neural Network", IEEE Conference March.2010 Indian Institute Of Science, Banglore , India. http://www.ijcaonline.org/archives/number3/8183. 